\title{
Relaxation behavior of nonlinear optical response in borate glasses containing gold nanoparticles
}

\author{
$\operatorname{AUTHOR}(\mathrm{S}):$
}

Sasai, Jun; Hirao, Kazuyuki

\section{CITATION:}

Sasai, Jun ...[et al]. Relaxation behavior of nonlinear optical response in borate glasses containing gold nanoparticles. JOURNAL OF APPLIED PHYSICS 2001, 89(8): 4548-4553

\section{ISSUE DATE:}

2001-04-15

URL:

http://hdl.handle.net/2433/39700

\section{RIGHT:}

Copyright 2001 American Institute of Physics. This article may be downloaded for personal use only. Any other use requires prior permission of the author and the American Institute of Physics. 


\title{
Relaxation behavior of nonlinear optical response in borate glasses containing gold nanoparticles
}

\author{
Jun Sasai ${ }^{\mathrm{a})}$ and Kazuyuki Hirao \\ Department of Material Chemistry, Graduate School of Engineering, Kyoto University, Sakyo-ku, Kyoto, \\ 606-8501, Japan
}

(Received 21 November 2000; accepted for publication 23 January 2001)

\begin{abstract}
The relaxation time of nonlinear optical response has been obtained by femtosecond pump-probe measurements for borate glasses containing gold nanoparticles prepared by conventional melt-quenching and heat treatment techniques. Size effects of gold nanoparticles on the relaxation process were investigated. The relaxation time of a fast relaxation component increases with a decrease of the particle radius. The tail of the decay curve due to a slow relaxation component increases with an increase of the particle size. These results indicate that the particle size is an important factor for relaxation processes. In addition, the tail component is very small and is not affected by the pump intensity. This shows that the borate glass matrix has superior properties in the slow relaxation process. (C) 2001 American Institute of Physics. [DOI: 10.1063/1.1355279]
\end{abstract}

\section{INTRODUCTION}

Glasses containing small metal particles have interesting linear and nonlinear optical properties which are quite different from those of bulk metals. They have a large optical absorption coefficient associated with the surface plasmon resonance of metal particles in the visible region, and show a large third-order nonlinearity around the absorption band. Their nonlinear response time is very short (about the order of $1 \mathrm{ps)}$ and these properties have attracted considerable attention since they have potential to be utilized as optical devices.

The relationship between the third-order nonlinear susceptibility $\chi^{(3)}$ and the fraction of particles is written as follows: ${ }^{1}$

$$
\chi^{(3)}=p\left|f_{i}(\omega)\right|^{2} f_{i}(\omega)^{2} \chi_{m}^{(3)},
$$

where $p$ is the volume fraction of metal particles in glass, $f_{i}(\omega)$ is the local field factor, and $\chi_{m}^{(3)}$ is the optical nonlinear susceptibility of the metal particle. This equation shows that $\chi^{(3)}$ is proportional to the fraction of metal particles. In order to increase the fraction of metal particles and $\chi^{(3)}$, these glasses have been prepared by various fabrication methods: melt quenching, ${ }^{2}$ ion implantation, ${ }^{3}$ ion exchange, ${ }^{4-7}$ sputtering, ${ }^{8-10}$ sol-gel, ${ }^{11}$ and others. ${ }^{12,13}$ On the other hand, the absorption coefficient is also proportional to the volume fraction of particle: ${ }^{1}$

$$
\alpha=p\left(\frac{\omega}{n c}\right)\left|f_{i}(\omega)\right|^{2} \epsilon_{m}^{\prime \prime} .
$$

Hence, the figure of merit is written as follows:

$$
\frac{\chi^{(3)}}{\alpha \tau} \sim \frac{p\left|f_{i}(\omega)\right|^{2} f_{i}(\omega)^{2} \chi_{m}^{(3)}}{p\left|f_{i}(\omega)\right|^{2} \epsilon_{m}^{\prime \prime}},
$$

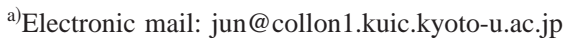

$$
\sim\left(\frac{f_{i}(\omega)^{2}}{\epsilon_{m}^{\prime \prime}}\right) \frac{\chi_{m}^{(3)}}{\tau}
$$

where $\epsilon_{m}=\epsilon_{m}^{\prime}+i \epsilon_{m}^{\prime \prime}$ is the dielectric constant of the metal particles, and $\tau$ is the relaxation time of the nonlinear optical response. This equation indicates that $\chi^{(3)} / \alpha \tau$ does not depend on the volume fraction of particles, $p$. It suggests that fabrication methods are not that important to increase the figure of merit. For the increase of the figure of merit, it is rather important to know how to change values of $f_{i}(\omega)$, $\epsilon_{m}^{\prime \prime}, \chi_{m}^{(3)}$, and $\tau$.

The particle size dependence of $f_{i}(\omega), \epsilon_{m}^{\prime \prime}$, and $\chi_{m}^{(3)}$ has been investigated, and the results show that $\chi^{(3)}$ increases as the particle size becomes larger in the case of gold, ${ }^{1}$ copper, ${ }^{2}$ and silver. ${ }^{2}$ As was shown above, many workers have investigated $\chi^{(3)}$ of these glasses and have come to know how to increase $\chi^{(3)} / \alpha$. Only a few workers, in contrast, have investigated the time resolved optical nonlinearity, that is, the measurement of $\tau$ for copper ${ }^{14,15}$ and gold ${ }^{16}$ nanoparticles by pump-probe measurement. These measurements enable us to know the mechanism and the relaxation dynamics of optical nonlinearity for metal nanoparticles system.

In this article, we report on the relaxation behavior of nonlinear optical response in the case of borate glasses containing gold nanoparticles obtained by femtosecond pumpprobe measurements. We examined the particle diameter dependence of the relaxation process.

\section{EXPERIMENT}

$73 \mathrm{~B}_{2} \mathrm{O}_{3} \cdot 25 \mathrm{Na}_{2} \mathrm{O} \cdot 2 \mathrm{Sb}_{2} \mathrm{O}_{3}$ glass doped with 0.5 mass $\%$ gold was prepared by means of a conventional meltquenching technique. Because the solubility of gold is very small, it was added as a solution of hydrochloric acid so that the gold was dispersed uniformly in the glass matrix. $\mathrm{Sb}_{2} \mathrm{O}_{3}$ was added to serve as a thermal reducing agent. The mixture was dried at $250{ }^{\circ} \mathrm{C}$ in a platinum crucible, and melted in an electric furnace at $1150{ }^{\circ} \mathrm{C}$ for $1 \mathrm{~h}$. The melt was quenched 


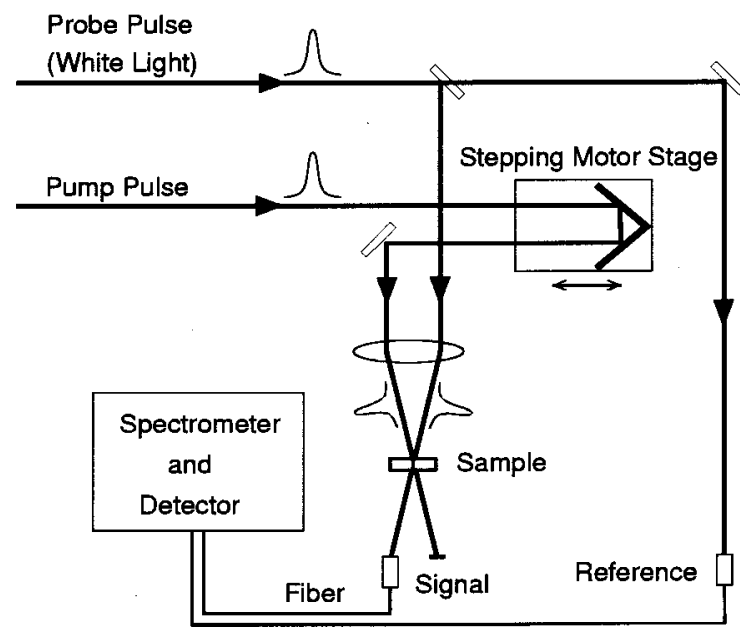

FIG. 1. Schematic illustration of the experimental setup for pump-probe measurement.

by being pressed between two iron plates to prevent nucleation of metal particles. After the resultant glass was annealed at $450{ }^{\circ} \mathrm{C}$ for $15 \mathrm{~min}$, it was cut into a plate $5 \mathrm{~mm}$ $\times 5 \mathrm{~mm} \times 1 \mathrm{~mm}$. The glass surfaces were polished with sandpaper and $\mathrm{CeO}_{2}$ powder.

These glass samples were heat treated above their glass transition temperature. Through this heat treatment process, reduction of gold ions occurred and metallic gold particles were precipitated in the glass matrix. Hereafter, the notations BN470, BN500, and BN530 are used for the samples heat treated at 470,500 , and $530^{\circ} \mathrm{C}$ for $9 \mathrm{~h}$, respectively.

Linear optical absorption spectra were measured by using a spectrophotometer. Transient absorption spectra were measured by means of the pump-probe method at room temperature. Figure 1 shows a schematic illustration of the setup for this measurement. In this measurement, we used an output pulse of an optical parametric amplifier (OPA) pumped by a $\mathrm{Ti}: \mathrm{Al}_{2} \mathrm{O}_{3}$ regenerative amplifier. The OPA pulse was used as a pump pulse. The pulse duration was about $150 \mathrm{fs}$. A white-light pulse generated through the self-phase modulation process in a water flow cell was used as a probe pulse, the pulse duration of which was also 150 fs. A differential absorption spectrum was obtained by subtracting the absorption spectrum with no pump pulse from that with the pump pulse at each delay time $\tau$, where $\tau$ is a time separation between the pump and probe pulse. The transmitted probe pulse and reference pulse were sent to a spectrometer by an optical fiber. Absorption change was detected by a $27.5 \mathrm{~cm}$ single monochromator with dual-diode arrays.

\section{RESULTS}

Absorption spectra of the BN470, BN500, and BN530 are shown in Fig. 2. The surface plasmon resonance is observed at around $2.35 \mathrm{eV}$ with a large shoulder due to the interband transition from the $d$ band to the Fermi level. As the heat treatment temperature becomes higher, the plasmon bandwidth becomes narrower.

Figure 3 shows the differential absorption $(\Delta \mathrm{OD})$ spectra of BN530 obtained by the femtosecond pump-probe mea-

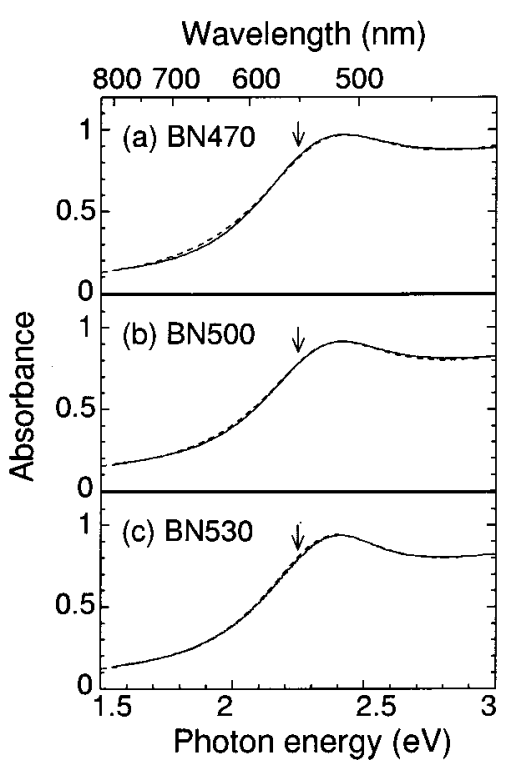

FIG. 2. Absorption spectra of (a) BN470, (b) BN500, and (c) BN530. Pump beam energies are indicated by arrows.

surement. The wavelength of the pump pulse is centered at $2.25 \mathrm{eV}(550 \mathrm{~nm})$. As the delay time increases, the magnitude of the bleaching becomes smaller. The bleaching is mostly recovered within $7 \mathrm{ps}$.

The time evolution of the bleaching signals of glasses at $2.3 \mathrm{eV}$ is shown in Fig. 4. Although the pump-pulse intensity is the same for all the samples, the maxima of $\triangle \mathrm{OD}$ are different. A tail component of the bleaching curve, which lasts for more than $20 \mathrm{ps,} \mathrm{exists} \mathrm{for} \mathrm{the} \mathrm{BN530,} \mathrm{while} \mathrm{it} \mathrm{is}$ not observed for BN470 and BN500.

Figure 5 shows the pump power dependence of the bleaching signal. As the pump power becomes larger, the magnitude of the bleaching signals becomes larger. The tail

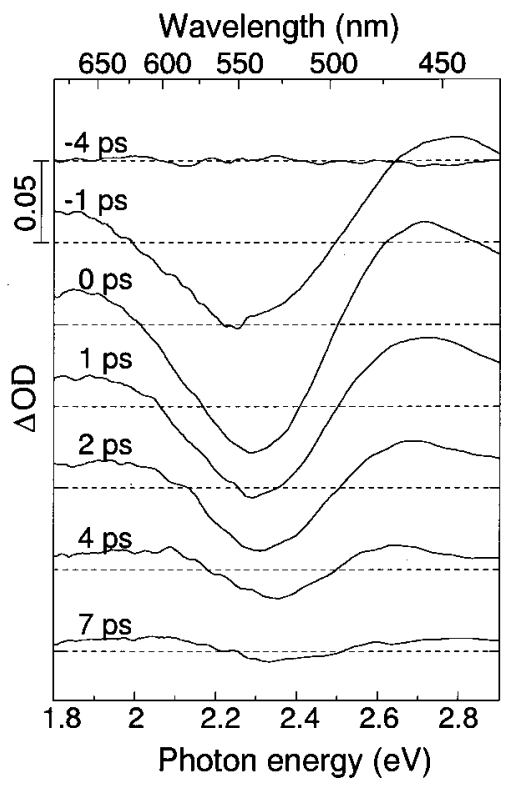

FIG. 3. Differential absorption spectra of BN530 at various delay times. 


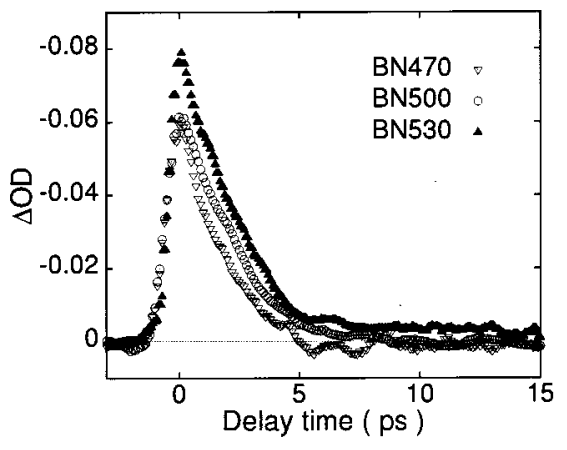

FIG. 4. Time evolution of the bleaching signals of BN470, BN500, and BN530 measured at $540 \mathrm{~nm}$ with laser intensity of $0.64 \mathrm{~mJ} \mathrm{~cm}^{-2}$.

component is not observed for any curves. These results suggest that the amplitude of the tail component does not depend on the incident pump power.

\section{DISCUSSION}

\section{A. Estimation of particle size by applying Mie theory}

In the present study, because of the low melting temperature of glass and low concentration of gold nanoparticles, it is technically difficult to observe the particle state by transmission electron microscopy. Therefore, in order to estimate the particle size, we must utilize another method. In the case of Ag nanoparticles, the correspondence of the particle diameter and plasmon resonance was reported. ${ }^{17}$ As for $\mathrm{Au}$ and $\mathrm{Cu}$ nanoparticles, since the plasmon and interband resonance bands overlap each other, it is difficult to estimate the plasmon bandwidth correctly. On the other hand, some researchers have reported the good coincidence of the particle size and the absorption spectra by applying the Mie scattering theory. ${ }^{14,18}$ Therefore, we regard theoretical fittings as the most reliable method by which to estimate particle size.

The absorption spectrum of glasses containing metal nanoparticles are fairly well reproduced by applying the Mie scattering theory: ${ }^{19}$

$$
\begin{aligned}
\alpha(\omega)= & \frac{2 \pi R^{2} L N}{x_{0}^{2}} \sum_{l=1}^{\infty}(2 l+1) \\
& \times \operatorname{Re}\left[a_{l}\left(x_{0}, x_{i}\right)+b_{l}\left(x_{0}, x_{i}\right)\right],
\end{aligned}
$$

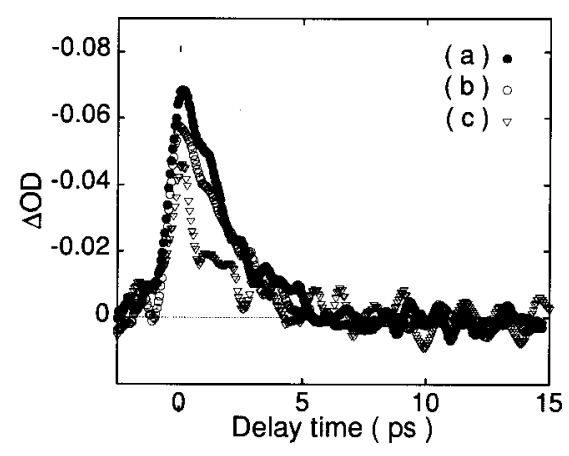

FIG. 5. Effect of the pump laser fluence on bleaching signals of BN500 measured at $540 \mathrm{~nm}$ with laser intensities of (a) 0.83 , (b) 0.51 , and (c) 0.32 $\mathrm{mJ} \mathrm{cm}{ }^{-2}$.
TABLE I. Running parameters of the theoretical fittings, particle diameter $R$ and damping constant $\hbar \gamma_{e e}$.

\begin{tabular}{ccc}
\hline \hline Sample & $\begin{array}{c}R \\
(\mathrm{~nm})\end{array}$ & $\begin{array}{c}\hbar \gamma_{e e} \\
(\mathrm{eV})\end{array}$ \\
\hline BN470 & 8.1 & 0.385 \\
BN500 & 8.5 & 0.370 \\
BN530 & 9.0 & 0.342 \\
\hline \hline
\end{tabular}

$$
x_{0}=\frac{R \omega}{c} \sqrt{\epsilon_{\text {glass }}}, \quad x_{i}=\frac{R \omega}{c} \sqrt{\epsilon_{\text {metal }}},
$$

where $R$ is the particle diameter, $L$ is the sample length, $N$ is the volume density of the nanoparticle, $c$ is the velocity of light, and $a_{l}$ and $b_{l}$ are the $l$ th Mie scattering coefficients, respectively. Here, the dielectric function of gold can be written follows; ${ }^{20}$

$$
\epsilon_{\text {metal }}=\epsilon_{\text {intra }}+\epsilon_{\text {inter }},
$$

where $\epsilon_{\text {intra }}$ is the Drude-like intraband term and $\epsilon_{\text {inter }}$ is the interband term, which are written respectively, as

$$
\epsilon_{\text {intra }}=1-\frac{\omega_{p}^{2}}{\omega\left(\omega+i \hbar \gamma_{\mathrm{eff}}\right)},
$$

and

$$
\begin{aligned}
\epsilon_{\text {inter }}= & K \int_{0}^{\infty} d x \frac{\sqrt{x-\omega_{g}}}{x}\left[1-F\left(x, \Theta_{e}\right)\right] \\
& \times \frac{\left(\omega^{2}-x^{2}-\gamma_{e e}^{2}-2 i \omega \gamma_{e e}\right)}{\left(\omega^{2}-x^{2}-\gamma_{e e}^{2}\right)^{2}+4 \omega^{2} \gamma_{e e}^{2}},
\end{aligned}
$$

where $\omega_{p \text {,eff }}$ is the effective plasma frequency of gold, $\gamma_{\text {eff }}$ is the effective plasma damping constant, $\hbar \omega_{g}$ is the gap energy, $F\left(x, \Theta_{e}\right)$ is the energy distribution function of electrons whose energy and temperature are $\hbar x$ and $\Theta_{e}$, respectively, and $\gamma_{e e}$ is the damping constant in the interband transition.

The plasma damping process is divided into two processes: surface and electron-phonon $(e-p)$ scattering. As for the e-p scattering, it depends linearly on the lattice temperature. Therefore, the following equation is derived: ${ }^{18}$

$$
\hbar \gamma_{e f f}\left(R, \Theta_{l}\right)=\frac{h V_{F}}{2 R}+\Gamma_{e-p} \Theta_{l},
$$

where $V_{F}$ is the Fermi velocity of gold, $\Theta_{l}$ is the lattice temperature, and $\Gamma_{\mathrm{e}-\mathrm{p}}$ is the electron-phonon damping constant, respectively. In this case, the electron mean free path is entirely limited by scattering at the surface of spheres. Therefore, the plasmon bandwidth is inversely proportional to the particle size.

The particle diameter can be estimated by applying this theory. The parameters used are $\epsilon_{\text {glass }}=2.31, L=0.10 \mathrm{~cm}$, $\hbar \omega_{p}=8.2 \mathrm{eV},{ }^{21}$ and $\hbar \omega_{g}=1.7 \mathrm{eV}$. ${ }^{1}$ Fitting results are shown in Fig. 2. As shown in Fig. 2, the absorption spectra are fairly well reproduced. The values of $R$ and $\gamma_{e e}$ estimated by theoretical fitting are shown in Table I. These results show that the particle size of gold nanoparticles increases with an increase of the heat treatment temperature. 


\section{B. Fast relaxation process}

For metal nanoparticle systems, it was suggested that their optical nonlinearity originates from an increase of the electron temperature caused by incidence of the laser beam. ${ }^{1,2,15,18,22}$ The incident laser pulse induces a nonequilibrium state, that is, the electron temperature is somewhat higher than the lattice temperature. The cooling process has been explained in terms of a thermal equilibrium process between the electron and lattice subsystems in a metal nanoparticle system $(\sim$ a few ps) as well as a thermal diffusion process from nanoparticles to the glass matrix $(\sim$ a hundred ps). ${ }^{2,18}$ That is, there are two components in the relaxation process. The relaxation time of each decay component is denoted by $\tau_{1}$ and $\tau_{2}$, respectively.

As for the fast component of the decay process, which is described by the electron-phonon ( $\mathrm{e}-\mathrm{p}$ ) coupling model, the energy flow between the electron and lattice subsystems is described as follows: ${ }^{23}$

$$
\begin{aligned}
& C_{e}\left(\Theta_{e}\right) \frac{\partial \Theta_{e}}{\partial t}=\kappa \nabla^{2} \Theta_{e}-G\left(\Theta_{e}-\Theta_{l}\right)+P(t), \\
& C_{1} \frac{\partial \Theta_{l}}{\partial t}=G\left(\Theta_{e}-\Theta_{l}\right),
\end{aligned}
$$

where $\kappa$ is the thermal conductivity of gold, $C_{e}$ and $C_{l}$ are the electron and lattice heat capacities, $G$ is the electronphonon coupling constant, and $P(t)$ is the absorbed laser power per unit volume. The first term on the right-hand side of Eq. (11) can be ignored because diffusive heat transport by electrons is negligible in the case of nanoparticle systems. Hence, the magnitude of $\mathrm{e}-\mathrm{p}$ coupling governs this process. A larger value of $G$ means strong coupling between the electron and lattice subsystems. In such a case, thermal equilibrium is quickly accomplished.

By assuming uniform electron and lattice temperature and small temperature dependence of physical parameters, the solution to the above equations as reported by Qiu and Tien $^{24}$ is

$$
\begin{aligned}
& \Theta_{e}-\Theta_{l}=\left(\Theta_{e 0}-\Theta_{l 0}\right) \exp \left(-t / \tau_{1}\right), \\
& \tau_{1}=\left(\frac{G}{C_{e}}+\frac{G}{C_{l}}\right)^{-1},
\end{aligned}
$$

where $\Theta_{e 0}$ and $\Theta_{l 0}$ are the initial electron and lattice temperatures, respectively. Considering the fact that $C_{l}$ is much larger than $C_{e}$ at room temperature, the equation is written as follows: $:^{24}$

$$
\tau_{1}=C_{e} / G \text {. }
$$

In this case, as shown in Table II, the relaxation time, $\tau_{1}$, of the BN470 is the shortest. In other words, the $G$ value of BN470 is largest. As for the size effects of $G$, Qiu and Tien reported that the $G$ value of gold thin film increases with a decrease of the film thickness since additional collisions of electrons are caused by a decrease of the atomic spacing and an increase of the influence of the grain boundaries and surfaces. ${ }^{24}$ Furthermore, Inouye et al. reported that the $G$ value of nanoparticle systems is larger than that of thin film systems because of a contribution by the e-p coupling
TABLE II. Relaxation time of the fast and slow decay processes and the initial temperature of the electron and lattice.

\begin{tabular}{ccccc}
\hline \hline Sample & $\begin{array}{c}\tau_{1} \\
(\mathrm{ps})\end{array}$ & $\begin{array}{c}\Theta_{e, 0} \\
\left({ }^{\circ} \mathrm{C}\right)\end{array}$ & $\begin{array}{c}\tau_{2, \text { calc }} \\
(\mathrm{ps})\end{array}$ & $\begin{array}{c}\Theta_{l, 0} \\
\left({ }^{\circ} \mathrm{C}\right)\end{array}$ \\
\hline BN470 & 1.83 & 1200 & 22.0 & $\ldots$ \\
BN500 & 2.36 & 1200 & 24.2 & $\ldots$ \\
BN530 & 2.31 & 1550 & 27.1 & 730 \\
\hline \hline
\end{tabular}

at the surface. ${ }^{18}$ These reports suggest that the $G$ value increases with an decrease of the particle size. Therefore, it is reasonable that the origin of the largest $G$ value of the BN470 is attributed to the smallest particle size.

Although the particle radius of the BN530 is larger than that of the BN500, their $\tau_{1}$ values are nearly equal to each other. This suggests that the particle size is not the only factor that determines the relaxation time. As shown in Fig. 4 , the maximum $\Delta \mathrm{OD}$ of the BN530 is larger than that of the other two specimens. The extent of the value of the maximum $\triangle \mathrm{OD}$ is different when the electron temperature is different. As shown in Fig. 6, the maximum electron temperatures of the BN470, BN500, and BN530 estimated by theoretical fitting are about 1550,1200 , and $1200^{\circ} \mathrm{C}$, respectively. This shows that the electron temperature of BN530 is higher than that of the others.

Therefore, as found from Eq. (15), the relaxation time becomes larger with an increase of the electron temperature. In the case of gold thin film, similar effects were observed: the higher electron temperature causes a larger value of $G,{ }^{25}$ and this results is similar to the measurements. Considering that the heat capacity of electrons linearly depends on the electron temperature in the real system, it is presumed that the higher electron temperature increases the heat capacity of electrons, and results in an increase of the apparent $G$ value. In this case, the higher electron temperature of the BN530 contributes to an increase of the apparent $G$ value, that is, a decrease of $\tau_{1}$ values, although the particle size of BN500 is smaller than that of the BN530. This causes the $\tau_{1}$ of the BN500 and BN530 to be nearly equal.

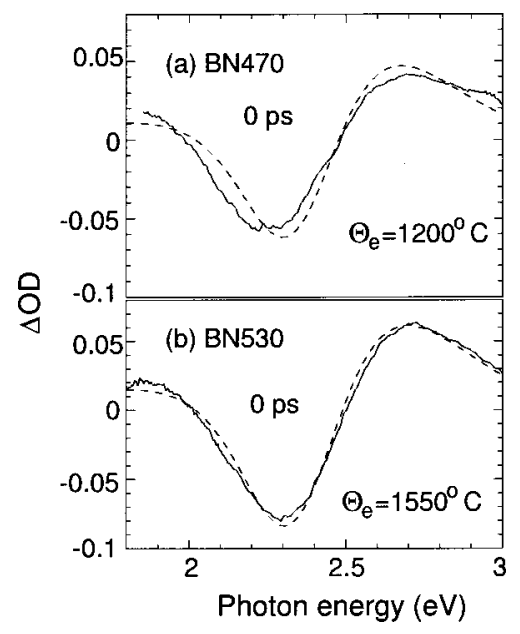

FIG. 6. Differential absorption spectra of (a) BN470 and (b) BN530 at 0 ps. Broken lines are theoretical fittings and the estimated values are $\Theta_{e}=$ (a) 1200 and (b) $1550{ }^{\circ} \mathrm{C}$, respectively. 


\section{Slow relaxation process}

As we mentioned above, the slow decay component of the relaxation process is caused by the thermal diffusion process from the nanoparticle to the glass matrix. Considering that the volume fraction of gold particles is very small, we can apply a quite simple model to describe this process. We assume that there is a gold nanoparticle in the glass matrix. The temperature in the particle is assumed to be uniform because the thermal diffusivity of gold is nearly $10^{3}$ times larger than that of glass. The thermal diffusion from a metal nanoparticle to the glass matrix is written as follows:

$$
\frac{\partial \Theta_{\text {glass }}}{\partial t}=D_{\text {glass }} \nabla^{2} \Theta_{\text {glass }},
$$

where $D_{\text {glass }}$ and $\Theta_{\text {glass }}$ are the thermal diffusivity and the temperature of the glass matrix, respectively. When we assumed that the temperature is continuous at the interface of the nanoparticle with the glass matrix, the energy-balance equation is given $b^{22}$

$$
C_{l} V_{0} \Theta_{l 0}=C_{l} V_{0} \Theta_{l}(t)+C_{\text {glass }} \int_{r}^{\infty} 4 \pi r^{2} \Delta \Theta_{\text {glass }}(t, r) d r,
$$

where $\Theta_{l 0}$ is the initial lattice temperature of the gold nanoparticle, $C_{\text {glass }}$ is the heat capacity of the glass, $\Delta \Theta_{\text {glass }}(t, r)$ $=\Theta_{\text {glass }}(t, r)-\Theta_{0}$ where $\Theta_{0}$ is the room temperature, and $V_{0}=4 \pi r^{3} / 3$. The lattice temperature inside the gold nanoparticle derived from the solution of these equations is ${ }^{18}$

$$
\Theta_{l}(t)=\frac{\Theta_{l 0}-\Theta_{0}}{1+3 \frac{C_{\text {glass }}}{C_{l}}\left\{\frac{\left(2 D_{\text {glass }} t\right)^{3 / 2}}{\sqrt{2 \pi} r^{3}} \exp \left(\frac{r^{2}}{4 D_{\text {glass }} t}\right) \operatorname{erfc}\left(\frac{r}{\sqrt{4 D_{\text {glass }} t}}\right)+\frac{2 D_{\text {glass }} t}{r^{2}}\right\}}+\Theta_{0} .
$$

Based on Eq. (18), we estimate the particle size dependence of the relaxation time, which is defined as the time when the temperature become $\Theta_{l 0} / e$, with the following values used: $D_{\text {glass }}=4.07 \times 10^{-2} \mathrm{~cm}^{2} \mathrm{~s}^{-1}$ and $C_{\text {glass }}=1.19$ $\times 10^{6} \mathrm{~J} \mathrm{~m}^{-3} \mathrm{~K}^{-1}$, which are the values of $75 \mathrm{~B}_{2} \mathrm{O}_{3} \cdot 25 \mathrm{Na}_{2} \mathrm{O}$. ${ }^{26}$ The relaxation times calculated for each sample are listed in Table II. By using these values, we can extrapolate the initial lattice temperature of the BN530, however that of the BN470 and BN500 cannot estimated because the tail component is barely observed. As for BN530, $\Theta_{l 0}$ is about $730^{\circ} \mathrm{C}$. Considering that the absorbance at the pump wavelength and the concentration of gold nanoparticles are nearly the same in all the samples, the initial lattice temperature of all the samples should be the same.

In previous work, it was revealed that all the absorbed energies through the interband transition and almost all absorbed energies by the surface plasmon are expended to heat up the lattice temperature. ${ }^{18}$ This result indicates that the larger laser fluence increases the absorbed energy used for the increase of lattice temperature.

Furthermore, when the laser fluence is larger than 1.0 $\mathrm{mJ} \mathrm{cm}{ }^{-2}$, the color of the irradiated spot disappears, that is, the absorbance due to the absorption band of gold nanoparticles decreases. The melting point of gold particles becomes lower with a decrease of particle size, ${ }^{27}$ and is estimated to be about $1000^{\circ} \mathrm{C}$. In addition, the glass matrix is also melted at about $800^{\circ} \mathrm{C}$. Therefore, in this case, the lattice temperature of gold nanoparticles or the temperature of the glass matrix close to gold nanoparticles becomes higher than their melting temperature. Afterward, the nanoparticles must be dissolved because the concentration of gold was smaller than its solubility. As a result, the lattice temperature is raised to at least $800^{\circ} \mathrm{C}$, which is consistent with the calculated initial lattice temperature $\Theta_{l, 0}$ and the results of previous work. ${ }^{18}$ Therefore, in this case, the tail of the decay curves is barely observed although the initial lattice temperature and the particle size are nearly the same as in the silica matrix system previously reported, which had a tail that lasted about 150 ps. ${ }^{18}$ In other words, it can be said that the borate glass matrix shows superior properties in the slow relaxation process.

\section{CONCLUSION}

We examined the optical nonlinear response for borate glass containing gold nanoparticles by femtosecond pumpprobe measurements. The temporal absorption change at the surface plasmon band shows that the electron-phonon coupling constant of gold nanoparticles decreases and the tail component of the decay curve increases as the particle radius increases. It indicates that a smaller particle diameter is desirable for the fast response of not only the fast component but also of the slow component. Furthermore, the tail component is barely observed and independent of laser fluence. It indicates that the borate glass matrix shows superior properties in the slow relaxation process.

\section{ACKNOWLEDGMENTS}

The authors would like to thank Dr. H. Inouye of the Institute of Applied Physics, University of Tsukuba, for pump-probe measurements and fruitful discussions and Dr. K. Tanaka of the Faculty of Engineering and Design, Kyoto Institute of Technology, for helpful discussions.

${ }^{1}$ F. Hache, D. Ricard, C. Flytzanis, and U. Kreibig, Appl. Phys. A: Solids Surf. A47, 347 (1988)

${ }^{2}$ K. Uchida, S. Kaneko, S. Omi, C. Hata, H. Tanji, Y. Asahara, A. J. Ikushima, T. Tokizaki, and A. Nakamura, J. Opt. Soc. Am. B 11, 1236 (1994)

${ }^{3}$ K. Fukumi, A. Chayahara, K. Kadono, T. Sakaguchi, Y. Horino, M. Miya, K. Fujii, J. Hayakawa, and M. Satou, J. Appl. Phys. 75, 3075 (1994). 
${ }^{4}$ N. Kitazawa, T. Yano, S. Shibata, and M. Yamane, Jpn. J. Appl. Phys., Part 2 33, L1245 (1994).

${ }^{5}$ H. Hofmeister, S. Thiel, M. Dubiel, and E. Schurig, Appl. Phys. Lett. 70, 1694 (1997)

${ }^{6}$ M. Ferrari, F. Gonella, M. Montagna, and C. Tosello, J. Appl. Phys. 79, 2055 (1996).

${ }^{7}$ N. Kitazawa, T. Yano, S. Shibata, and M. Yamane, Jpn. J. Appl. Phys., Part 1 35, 2228 (1996).

${ }^{8}$ I. Tanahashi, Y. Manabe, T. Tohda, S. Sasaki, and A. Nakamura, J. Appl. Phys. 79, 1244 (1996).

${ }^{9}$ I. Tanahash, M. Yoshida, T. Tohda, S. Sasaki, T. Tokizaki, and A. Nakamura, Jpn. J. Appl. Phys., Part 2 33, L1410 (1994).

${ }^{10}$ I. Tanahashi, M. Yoshida, T. Manabe, and T. Tohda, J. Mater. Res. 10, 362 (1995)

${ }^{11}$ I. Tanahashi and T. Tohda, J. Am. Ceram. Soc. 79, 796 (1996).

${ }^{12}$ T. Noguchi, S. Hayashi, M. Kawahara, K. Gotoh, Y. Yamaguchi, and S. Deki, Appl. Phys. Lett. 62, 1769 (1993).

${ }^{13}$ A. Berger and H. Hofmeister, Nanostruct. Mater. 3, 53 (1993).

${ }^{14}$ J. Y. Bigot, J. C. Merle, O. Cregut, and Deunois, Phys. Rev. Lett. 75, 4702 (1995).

${ }^{15}$ T. Tokizaki, A. Nakamura, S. Kaneko, K. Uchida, S. Omi, H. Tanji, and Y. Asahara, Appl. Phys. Lett. 65, 941 (1994).
${ }^{16}$ M. Perner, P. Bost, U. Lemmer, G. von Plessen, J. Feldmann, U. Becker, M. Mennin, M. Schmitt, and H. Schmidt, Phys. Rev. Lett. 78, 2192 (1997).

${ }^{17}$ W. P. Halperin, Rev. Mod. Phys. 58, 533 (1986).

${ }^{18}$ H. Inouye, K. Tanaka, I. Tanahashi, and K. Hirao, Phys. Rev. B 57, 11334 (1998).

${ }^{19}$ H. C. van de Hulst, Light Scattering by Small Particles (Dover, New York, 1981).

${ }^{20}$ R. Ruppin, J. Appl. Phys. 59, 1355 (1986).

${ }^{21}$ S. Suárez, W. E. Bron, and T. Juhasz, Phys. Rev. Lett. 75, 4536 (1995).

${ }^{22}$ M. J. Boemer, J. W. Haus, and P. R. Ashley, J. Opt. Soc. Am. B 7, 790 (1990)

${ }^{23}$ S. I. Anisimov, B. L. Kapeliovich, and T. L. Perel'man, Sov. Phys. JETP 39, 375 (1974).

${ }^{24}$ T. Q. Qiu and C. L. Tien, Trans. ASME, Ser. C: J. Heat Transfer 115, 842 (1993)

${ }^{25}$ W. S. Fann, R. Storz, H. W. K. Tom, and J. Bokor, Phys. Rev. Lett. 68, 2834 (1992)

${ }^{26}$ O. V. Mazurin, M. V. Streltsina, and T. P. Shavaiko-Shvaikonskaya, Handbook of Glass Data (Elsevier, New York, 1985), Part B.

${ }^{27}$ P. Buffat and J.-P. Borel, Phys. Rev. A 13, 2287 (1976). 